\title{
"Health in all policies" perspectives highlight the trade-offs that governments make when buying population health
}

\section{L'optique de "la santé dans toutes les politiques » met en évidence les compromis des gouvernements lorsqu'ils achètent la santé des populations}

\author{
Daniel Dutton ${ }^{1}$
}

Published online: 19 February 2020

(C) The Canadian Public Health Association 2020

Dr. Paul Kershaw's (2020) thought-provoking piece focuses on the distribution of government spending across age groups and how inequitably that distribution has evolved over time. By comparing those aged 65 and over with those 45 and under, he sets up a longitudinal comparison of spending on healthcare and various social benefits. There is a large imbalance in health and social spending between the older and younger groups, driven in large part by health spending increases for the older group.

The increase in health spending is perhaps expected by observers, as Canada has more seniors now than in 1976 (the starting year for Kershaw's analysis). In response, policymakers have taken some fiscally prudent action like changing the Canada Pension Plan from a pay-as-you-go format to prepaid. Healthcare, however, remains funded by general revenue, which means two things: first, that increasing health spending crowds out other uses of the money; and second, that the older group is consuming a larger share of total resources over time in the form of health spending. Therein lies the problem. Ironically, increased funding to the healthcare system for the older group is detrimental to population health by crowding out childcare, family income support, or parental leave, all of which have the potential to drive health outcomes themselves.

Canadian rhetoric on healthcare spending centres around the notion that health outcomes are determined primarily by

Daniel Dutton

Daniel.Dutton@dal.ca

1 Department of Community Health and Epidemiology, Dalhousie University, Halifax, NS, Canada medical care. We see this during elections or budget discussions, where decisions around how much to spend on which aspects of the healthcare system are presented alongside concerns about the fate of those who rely on said system. Kershaw invokes the literature showing that multiple government spending portfolios beyond healthcare can influence health outcomes, advocating that a "health in all policies" perspective allows us to explicitly acknowledge that actions on nonhealth spending can have health consequences.

The importance of evaluating policies jointly with a "health in all policies" perspective is the ability to identify health gains from non-health spending. The phenomenon of incorrectly labeling gains to population health as mostly attributable to healthcare spending is common enough to have a name: the wrong pockets problem. Population health benefits arise from many interventions that we incorrectly treat as cost-outflows or potential targets for low-consequence cost-cutting. Interventions like subsidized housing, social assistance payments, or child benefits are all non-health spending interventions with substantial health benefits. If we explicitly adopt a "health in all policies" perspective, we reduce the risk of contributing to the wrong pockets problem by overlooking these benefits when considering the value of interventions.

Our society's spending on seniors is, of course, not a bad thing. Canada has been successful in controlling poverty among seniors and is sometimes considered a world leader in decreasing seniors' poverty prevalence. Medical care advances in Canada continue to improve the quality and quantity of life for those who receive them. People tend to use more healthcare services in their final year of life than at any other point, which makes medical care for older individuals more expensive on average. Kershaw's succinct analysis forces us 
to consider the sacrifices we have made to afford this. Was the substantial enrichment of health and pension spending on seniors worth the relatively paltry increases for those less than 45 years old? The answer to that question requires a value judgement on the relative importance of targeting seniors as opposed to, say, children.

The Canadian obsession with targeted interventions is creating groups who are left behind. These groups are, in other words, the untargeted. An example of non-health spending targeting non-seniors is the Canada Child Benefit, which is held up as an effective poverty-fighting intervention aimed at families with children. Certainly, this is a welcome contribution to tipping the spending scales in favour of the younger age group and toward more social support, yet it does nothing for those without children. A group rarely on the receiving end of targeted interventions in Canada, for instance, are those at risk of becoming homeless, who are overwhelmingly male, single, and middle-aged. Once an individual becomes homeless, he or she is eligible for many social programs, but homelessness is an outcome best avoided altogether! Similarly, there are many low-income 64-year-olds who we insist must survive to age 65 whereupon they qualify for old age security payments, which are more generous than provincial social assistance payments in most cases. Low-income couples without children are similarly ignored.

Targeted interventions have their appeal in their perceived efficiency at directing resources to those who we identify as worthy recipients. Universal interventions could hold promise for improving population health more equitably but are potentially less politically feasible. Geoffrey Rose's paradox of prevention (Rose et al. 2008) is the idea that small changes in risk to a large group of people can manifest as substantial population health improvements, but individuals (rightly) see these small changes in their own risk as insubstantial and perhaps irrelevant. Targeted and universal interventions can co-exist to address separate issues, and do in the Canadian context, but policy action has great resonance when aimed at a target deserving of such action. Thus, spending on seniors or children directly makes intuitive sense if those are the groups we feel deserve to live in improved environments. It is unlikely that we face a zero-sum game in deciding whether to spend our marginal dollars on children or seniors, and universal policies that address equity issues could be the missing piece of the conversation. Kershaw's work is a driving force behind evaluating those policy packages that affect entire sections of society and considering, in turn, their joint efficiency in generating outcomes we value, like health.

So, in the face of this funding inequity precedent, what is a policymaker to do? Kershaw identifies recalibrating funding decisions based on a "health in all policies" perspective to benefit younger Canadians, with specific examples pertaining to national pharmacare and physician remuneration, indicating prepayment for the former and control of the latter as favourable for maintaining a high social-to-health spending ratio. Trudeau's Liberals recently campaigned on more money to childcare outside school hours and raising the Canada Child Benefit, both of which are targeted interventions to help families with children present. We need to be flexible in how we think about who deserves to benefit from social spending, or in other words: whose health counts? We need to be imaginative in how we conceive of health service delivery. Can health care system redesign improve Canada's international ranking in health care efficiency? Is it enough to propose more of the same kind of interventions, or is it time to consider more radical changes to health and social program delivery? Is the tax system an appropriate vehicle for delivering these types of benefits to families when we know that low-income families are the least likely to file taxes? Kershaw shows us that the evolution of spending in the past four decades is a poor guide to answering these questions. Similarly, it is not clear that further enrichment of targeted interventions will achieve an equitable balance.

\section{Éditorial}

L'article fascinant de Paul Kershaw (Kershaw 2020) porte sur la distribution des dépenses publiques entre les tranches d'âge et sur l'évolution inégale de cette distribution au fil du temps. En comparant les personnes de 65 ans et plus à celles de 45 ans et moins, il établit une comparaison longitudinale des dépenses consacrées aux soins de santé et à divers avantages sociaux. Il existe un grand déséquilibre dans les dépenses sociales et de santé pour le groupe le plus âgé et le groupe le plus jeune, principalement en raison de l'augmentation des dépenses de santé pour le premier groupe.

L'augmentation des dépenses de santé n'est pas une surprise pour les observateurs, car le Canada compte plus d'aînés aujourd'hui qu'en 1976 (première année de l'analyse de Kershaw). En réaction, les responsables des politiques ont pris des mesures prudentes sur le plan financier, comme de changer le mode de financement par répartition du Régime de pensions du Canada pour un régime entièrement capitalisé. Les soins de santé, par contre, sont encore financés par les recettes générales, ce qui signifie deux choses : d'abord, que la hausse des dépenses de santé évince d'autres utilisations des fonds; et ensuite, que le groupe le plus âgé consomme une plus grande part des ressources totales au fil du temps sous forme de dépenses de santé. C'est là où le bât blesse. Curieusement, le financement accru du système de soins de santé en faveur du groupe le plus âgé nuit à la santé des populations, car il évince les dépenses pour les services de garde, le soutien du revenu familial ou les congés parentaux, qui ont toutes pourtant le potentiel d'influer sur les résultats de santé.

Le discours canadien sur les dépenses de soins de santé repose sur la notion que les résultats de santé sont 
principalement attribuables aux soins médicaux. Nous le voyons en période électorale et dans les discussions budgétaires, où les décisions sur les sommes à dépenser pour tel ou tel aspect du système de soins de santé sont couplées aux inquiétudes sur le sort des personnes qui dépendent dudit système. La littérature scientifique le montre : beaucoup d'autres portefeuilles de dépenses publiques que celui des soins de santé peuvent influer sur les résultats de santé, et Kershaw fait valoir qu'une optique de « santé dans toutes les politiques » nous permettrait de reconnaître explicitement que les mesures axées sur des dépenses non sanitaires peuvent aussi avoir des conséquences pour la santé.

Il est important d'évaluer les politiques en les arrimant à une optique de « santé dans toutes les politiques », car cela permet de repérer les gains découlant des dépenses non sanitaires. Le phénomène qui consiste à attribuer principalement les gains de santé des populations aux dépenses de soins de santé est si courant qu'il a un nom : le problème des « mauvaises poches ». Les avantages pour la santé des populations découlent de nombreuses interventions qui sont traitées à tort comme des sorties de trésorerie ou comme les cibles possibles de mesures de réduction de coûts que nous jugeons être sans conséquence. Des interventions comme les logements subventionnés, les paiements d'aide sociale ou les prestations pour enfants impliquent toutes des dépenses non sanitaires, mais qui comportent des avantages considérables sur le plan de la santé. En adoptant explicitement une optique de «santé dans toutes les politiques », nous réduisons le risque d'accentuer le problème des mauvaises poches en négligeant les avantages de ces interventions dans notre analyse de leur valeur.

Bien entendu, les dépenses sociales pour nos aînés ne sont pas une mauvaise chose. Le Canada lutte efficacement contre la pauvreté chez les personnes âgées; il est même parfois considéré comme un chef de file mondial en matière de réduction de la prévalence de la pauvreté chez les aînés. Les avancées médicales au pays continuent d'améliorer la qualité et la durée de la vie des personnes qui en sont les bénéficiaires. Les gens ont tendance à utiliser plus de services de santé au cours de leurs dernières années de vie qu'à tout autre moment, ce qui rend les soins médicaux aux personnes âgées plus chers en moyenne. L'analyse succincte de Kershaw nous force à tenir compte des sacrifices que nous avons faits pour nous payer ces améliorations. L'enrichissement considérable des dépenses pour la santé et des rentes aux aînés en vaut-il la peine lorsqu'on le compare aux augmentations dérisoires destinées aux moins de 45 ans? La réponse à cette question nécessite un jugement de valeur quant à l'importance relative de cibler les aînés plutôt que les enfants, par exemple.

Avec l'obsession canadienne pour les interventions ciblées, certains groupes sont laissés pour compte. Ces groupes, autrement dit, sont les « non ciblés ». L'Allocation canadienne pour enfants, une dépense non sanitaire qui ne cible pas les aînés, est donnée en exemple d'une intervention efficace de lutte contre la pauvreté axée sur les familles avec enfants. Elle est certainement souhaitable pour faire pencher la balance des dépenses en faveur de la tranche d'âge la plus jeune et pour accroître le soutien social, mais elle ne fait rien pour les personnes sans enfants. L'un des groupes qui est rarement bénéficiaire d'interventions ciblées au Canada, par exemple, est celui des personnes à risque de sans-abrisme, qui sont en très grande majorité des hommes célibataires d'âge moyen. Lorsqu'une personne se retrouve dans la rue, elle devient admissible à une foule de programmes sociaux, mais le sansabrisme est un résultat à éviter! De même, nombreuses sont les personnes de 64 ans à faible revenu que nous insistons pour faire attendre jusqu'à 65 ans avant de leur donner droit aux prestations de Sécurité de la vieillesse, plus généreuses que la plupart des paiements d'aide sociale provinciaux. Les couples à faible revenu sans enfants sont négligés eux aussi.

Les interventions ciblées ont leur intérêt, car elles semblent orienter efficacement les ressources vers les personnes que nous jugeons méritoires. Les interventions universelles ont beau être prometteuses pour améliorer plus équitablement la santé des populations, elles sont potentiellement moins faisables sur le plan politique. Le paradoxe de la prévention de Geoffrey Rose (Rose 2008) est l'idée selon laquelle les petits changements du niveau de risque d'un grand groupe de personnes peuvent entraîner des améliorations considérables de la santé des populations, mais les gens considèrent (à raison) que ces petits changements de leur propre niveau de risque sont négligeables, voire non pertinents. Des interventions ciblées et universelles peuvent coexister pour aborder des problèmes distincts, ce qui se fait déjà dans le contexte canadien, mais l'action politique trouve plus d'écho lorsqu'elle cible un groupe jugé mériter cette action. Il semble donc logique de dépenser directement pour les aînés ou les enfants si ce sont ces groupes-là qui méritent selon nous de vivre dans de meilleures circonstances. Il est peu probable que la décision de dépenser nos dollars marginaux pour les enfants ou pour les aînés fasse nécessairement des gagnants et des perdants; les politiques universelles qui abordent les problèmes d'équité pourraient donc être la pièce manquante de la conversation. L'article de Kershaw nous incite à évaluer les ensembles de mesures qui touchent des pans entiers de la société et à déterminer l'efficacité conjointe de ces mesures pour produire des résultats qui ont de la valeur à nos yeux, comme la santé.

Face à ce précédent de financement inéquitable, que peut faire une ou un responsable des politiques? Kershaw propose de réorienter les décisions de financement selon une optique de « santé dans toutes les politiques » en faveur des Canadiens les plus jeunes et donne des exemples précis afférents à l'assurance-médicaments nationale et à la rémunération des médecins; le paiement par anticipation de la première et le contrôle de la seconde contribueraient selon lui au maintien 
d'un ratio élevé de dépenses sociales par rapport aux dépenses de santé. Les libéraux de Justin Trudeau ont fait campagne dernièrement en faveur d'un financement plus généreux des services de garde en dehors des heures de cours et d'une hausse de l'Allocation canadienne pour enfants, deux interventions ciblées pour aider les familles avec enfants. Nous devons nous montrer souples dans notre façon de déterminer qui mérite de bénéficier des dépenses sociales, ou autrement dit qui a droit à la santé. Nous devons faire preuve d'imagination dans notre façon de concevoir la prestation des services de santé. La refonte du système de soins de santé peut-elle améliorer le classement international du Canada en matière d'efficacité des soins de santé? Est-il suffisant de proposer plus d'interventions du même acabit, ou est-il temps d'envisager des changements plus radicaux à la prestation des programmes sociaux et de santé? Le système fiscal est-il le moyen indiqué pour procurer ce genre d'avantages aux familles, sachant que les familles à faible revenu sont les moins susceptibles de produire des déclarations de revenus? Kershaw nous montre que l'évolution des dépenses depuis 40 ans n'est pas un indicateur fiable pour répondre à ces questions. De même, il n'est pas clair si un enrichissement plus poussé des interventions ciblées redressera équitablement la balance.

\section{References/Références bibliographiques}

Kershaw, P. (2020). A "health in all policies" review of Canadian public finance. Canadian Journal of Public Health, 111(1). https://doi.org/ 10.17269/s41997-019-00291-4.

Rose, G., Khaw, K.-T., \& Marmot, M. (2008). Rose's strategy of preventive medicine. Oxford University Press.

Publisher's note Springer Nature remains neutral with regard to jurisdictional claims in published maps and institutional affiliations. 